ВІСНИК

ОДЕСЬКОГО НАЦІОНАЛЬНОГО

МОРСЬКОГО УНІВЕРСИТЕТУ
HERALD

OF THE ODESSA NATIONAL

MARITIME UNIVERSITY

№ $3(63), 2020$

УДК 629.5.052.3-52-049.5

DOI 10.47049/2226-1893-2020-3-106-117

\title{
ИСПОЛЬЗОВАНИЕ ТЕХНОЛОГИЙ РЕЧНЫХ ИНФОРМАЦИОННЫХ СИСТЕМ ДЛЯ УЧЕТА ДИНАМИКИ РЕЛЬЕФА ДНА
}

\section{В.В. Шпилевский}

аспирант, капитан

Национальный университет «Одесская морская академия», Одесса, Украина

Аннотация: Любое исследование предпочтительно проводить с учетом изменения состояния объекта во времени и под воздействием различных факторов одновременно и динамически. В статье динамические прочессы делятся на «значимые» $и$ «несущественные». Эта зависимость определяется масштабом пространства и времени, где и когда они происходят. Понятие «маситабный фактор» вводится с иелью определения степени значимости влияния динамических процессов при проведении исследований.

Ключевые слова: РИС, динамические прочессы, «scale factor», «chart dynamic model», ЭКДИС.

УДК 629.5.052.3-52-049.5

DOI 10.47049/2226-1893-2020-3-106-117

\section{ВИКОРИСТАННЯ ТЕХНОЛОГІЙ РІЧКОВИХ ІНФОРМАЦЙНИХ СИСТЕМ ДЛЯ ОБЛІКУ ДИНАМІКИ РЕЛЬЕФУ ДНА}

\section{В.В. Шпилевський} аспірант, капітан

Національний університет «Одеська морська академія», Одеса, Украӥна

Анотація: Будь-яке дослідження переважно проводити з урахуванням зміни стану об'єкта в часі і під впливом різних факторів одночасно і динамічно. У статті динамічні процеси діляться на «значущі» $i$ «несуттєві». Ця залежність визначається масштабом простору $i$ часу, де і коли вони відбуваються. Поняття «масштабний фактор» вводиться з метою визначення ступеня значущості впливу динамічних процесів при проведенні досліджень.

Ключові слова: PIC, динамічні прочеси, «scale factor», "chart dynamic model», ЕКДІС.

(C) Шпилевский В.В., 2020 
UDC 629.5.052.3-52-049.5

DOI 10.47049/2226-1893-2020-3-106-117

\title{
TAKING INTO ACCOUNT THE DYNAMICS OF THE BOTTOM RELIEF FOR TECHNOLOGIES RIS
}

\author{
V. Shpylevsky \\ PhD student, Captain \\ National University «Odessa Maritime Academy», Odessa, Ukraine
}

Abstract. Any study is preferable to perform taking into account changes in the state of the object in time and under the influence of various factors simultaneously and dynamically. The article divides dynamic processes into "significant» and «insignificant». This dependence is determined by the scale of the space and time where and when they occur. The concept of "scale factor» is introduced in order to determine the degree of significance of the influence of dynamic processes when performing research.

Keywords: RIS, dynamic processes, "scale factor», "chart dynamic model», ECDIS.

Постановка проблемы. Все, что нас окружает, находится в постоянном движении в той или иной системе координат. Навигационные карты, входящие в систему Речных Информационных Систем (РИС), не являются исключением. В работах $[1 ; 2 ; 3]$ представлены факторы, влияющие на изменение состояния грунтов в различных условиях природного ландшафта и даны описания последствий. Однако рекомендации по комплексному учету характеристик воздействия отсутствуют. Рассмотрим в процессе нашего исследования пути решения задачи учета динамики рельефа морского дна на тех участках и на тех временных интервалах, где это ощутимо в процессе навигации.

Целью работы является построение математической модели на участках интенсивного изменения рельефа дна, что позволит в дальнейшем избежать негативных последствий при движении судов на этих участках.

Задачей исследования является определение качественных и количественных характеристик процесса динамики подводного рельефа дна. Совместный учет предпосылок, последствий и реального состояния грунта возможно отобразить на экранах приборов с применением РС [5].

В качестве обоснования, выбранного для исследования участка, ниже представлены изображения результатов повторных промеров в разные промежутки времени [7]. Здесь можно видеть как изменяют свое положение изобаты с течением времени с интервалом в полгода. Исследования проводились на участке ГСХ «Быстрое», где после сильных и продолжительных штормов рельеф дна может ощутимо поменять свой рисунок. 
ВІСНИК

ОДЕСЬКОГО НАЦІОНАЛЬНОГО

МОРСЬКОГО УНІВЕРСИТЕТУ

№ 3 (63), 2020
HERALD

OF THE ODESSA NATIONAL

MARITIME UNIVERSITY

№ 3 (63), 2020

Фрагменты динамической карты представлены на рис. 1 a, b, с, где показано изображение изобат на исследуемом полигоне в различные периоды времени.

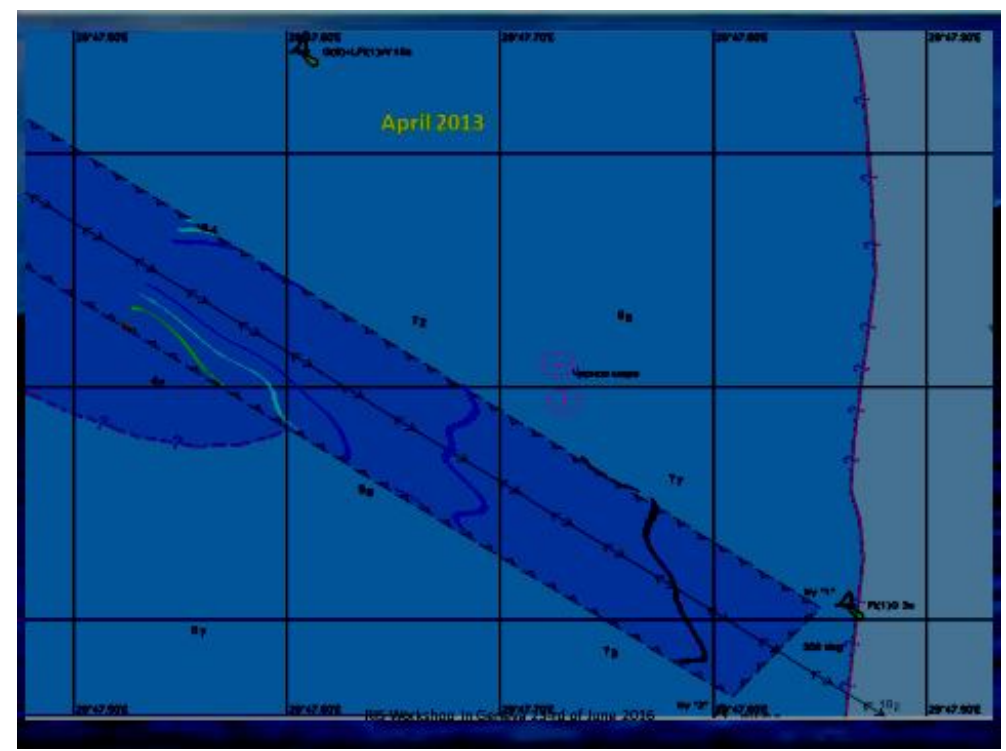

Рис. 1 а. Электронная навигационная карта в апреле 2013 г.

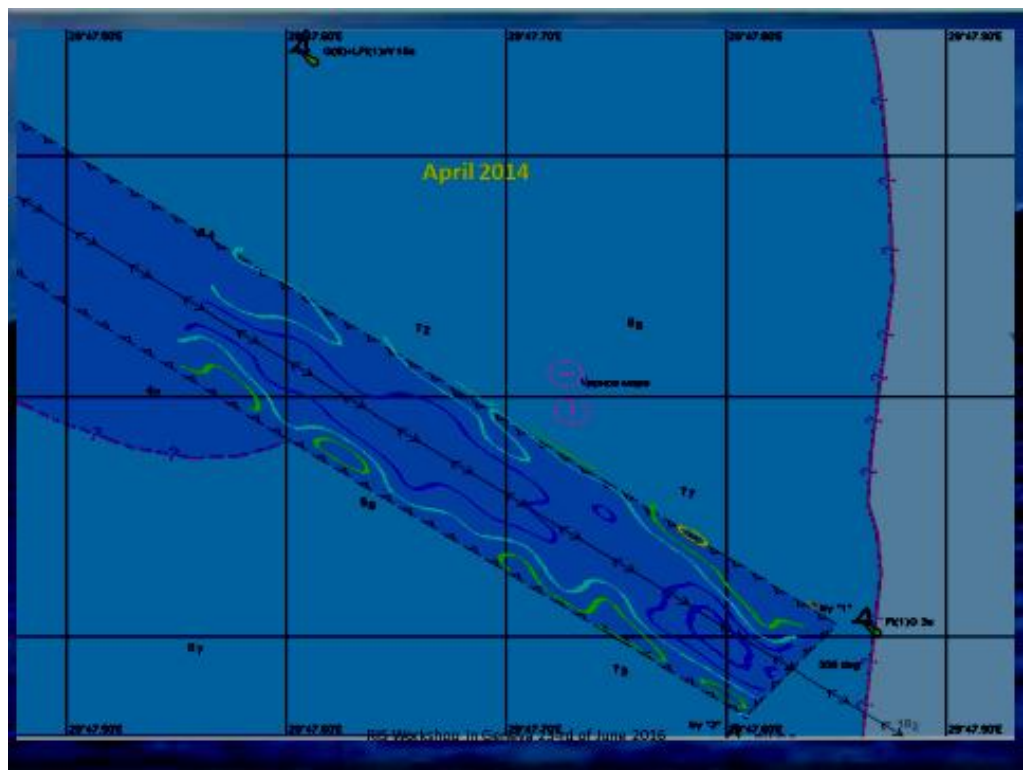

Рис. 1 b. Электронная навигационная карта в апреле 2014 г. 
ВІСНИК

ОДЕСЬКОГО НАЦІОНАЛЬНОГО

МОРСЬКОГО УНІВЕРСИТЕТУ

№ 3 (63), 2020
HERALD

OF THE ODESSA NATIONAL

MARITIME UNIVERSITY № 3 (63), 2020

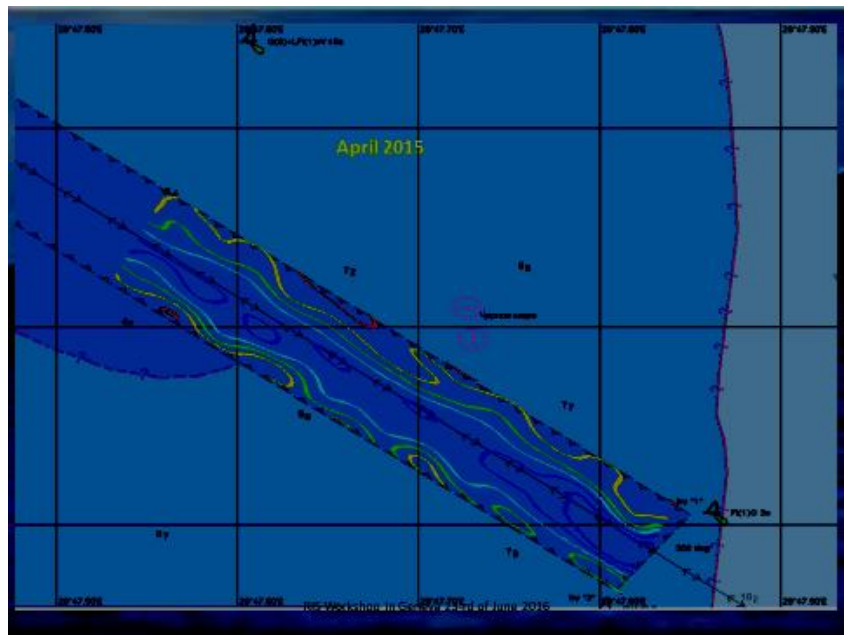

Рис. 1 с. Электронная навигационная карта в апреле 2015 2.

При рассмотрении модели движения подводного рельефа, судна и водной поверхности следует учесть, что все эти движения разномасштабные во времени. Так, например, рассмотрим наиболее часто применяемые модели движения судна.

Математическая модель движения судна как управляемой динамической системы в общем случае может быть представлена следующим образом:

$$
S(t)=F(t, C, S(0), U(t), L(t), E(t)),
$$

где $F$ - оператор, характеризующий данную конкретную математическую модель;

$C$ - вектор постоянных параметров системы, которые характеризуют данное конкретное моделируемое судно;

$S(t)$ - совокупность переменных параметров, описывающих состояние системы в момент времени $t$. Если рассматривать плоскопараллельное движение судна, можно ограничиться тремя параметрами координат $x_{0}$ и $y_{0}$ и курсовым углом $q$

$$
S(t)=\left(x_{0}(t), y_{0}(t), q(t)\right)
$$

$U(t)$ - управляющие воздействия на систему в разные моменты времени: угол перекладки руля $\delta R(t)$, частота вращения $n m(t)$ и шаговое отношение $H / D(t)$ гребного винта, положение регулятора подруливающего устройства $N$ ПУотн $(t)$, который задает его относительную мощность в процентах от максимально возможной

$$
U(t)=\delta R(t), n m(t), \frac{H}{D(t)}, N \Pi \text { Уотн }(t) .
$$


$L(t)$ - функция нагрузки на систему, в данном случае распределение всех грузов на судне;

$E(t)$ - функция внешних возмущающих воздействий на систему: глубины во всех точках акватории, скорости и направления ветра и течения, амплитудный и фазовый спектр волнения, а также спектр направлений распространения волн по всем частотам для всех точек акватории во все моменты времени.

Существующие математические модели плоскопараллельного движения судна могут быть представлены в форме, которая в более развернутом виде выглядит так:

$$
\begin{gathered}
\frac{d_{2} x_{0}}{d t_{2}}=i a F x_{0 i} \frac{t, C, \frac{d x_{0}}{d t}, \frac{d y_{0}}{d t}, \frac{d q}{d t}, x_{0}(t), y_{0}(t), q(t), U(t), L(t), E(t)}{m} \\
\frac{d_{2} y_{0}}{d t_{2}}=i \sum F y_{0 I} \frac{t, C, \frac{d x_{0}}{d t}, \frac{d y_{0}}{d t}, \frac{d q}{d t}, x_{0}(t), y_{0}(t), q(t), U(t), L(t), E(t)}{m} \\
\frac{d q}{d t}=i \sum M_{i} \frac{t, C, \frac{d x_{0}}{d t}, \frac{d y_{0}}{d t}, \frac{d q}{d t}, x_{0}(t), y_{0}(t), q(t), U(t), L(t), E(t)}{I_{z}}
\end{gathered}
$$

Однако большинством авторов используется следующая более удобная эквивалентная структура математической модели:

$$
\left\{\begin{array}{l}
\frac{d x_{0}}{d t}=v \cos (q-\beta) ; \\
\frac{d y_{0}}{d t} v \sin (q-\beta) ; \\
\frac{d q}{d t}=w ; \\
\frac{d v}{d t}=-v w \sin \beta \cos \beta\left(\frac{1}{1+k_{11}}-\frac{1}{1+k_{22}}\right)-\frac{\left(i \sum F_{y i}\right) \sin \beta}{\left(1+k_{22}\right) \rho V}+\frac{\left(i \sum F_{y i}\right) \cos \beta}{\left(1+k_{11}\right) \rho V} ; \\
\frac{d \beta}{d t}=w\left(\frac{\sin ^{2} \beta}{1+k_{11}}+\frac{\cos ^{2} \beta}{1+k_{22}}\right)-\frac{\left(i \sum F_{y i}\right) \sin \beta}{\left(1+k_{11}\right) \rho V v}+\frac{\left(i \sum F_{y i}\right) \cos \beta}{\left(1+k_{22}\right) \rho V v} ; \\
\frac{d w}{d t}=\frac{i \sum M_{i}}{\left(1+k_{66}\right) I_{z}} ; \\
i \sum F_{x i}\left(t, C, v(t), w(t), \beta(t), x_{0}, y_{0}(t), q(t), U(t), L(t), E(t)\right)=X_{6}+X_{p}+T_{E}+X_{\text {внеи } ;} ; \\
i \sum F_{y i}\left(t, C, v, w(t), \beta(t), x_{0}, y_{0}(t), q(t), U(t), L(t), E(t)\right)=Y_{6}+Y_{p}+Y_{\text {Пу }}+Y_{\text {внеи }} ; \\
i \sum M_{i}\left(t, C, v(t), w(t), \beta(t), x_{0}, y_{0}(t), q(t), U(t), L(t), E(t)=M_{в}+M_{p}+M_{\text {Пу }}+M_{\text {внеи }} ;\right.
\end{array}\right.
$$


Здесь $v(t)$ и $w(t)$ - линейная и угловая скорость судна;

$\beta(t)$ - угол дрейфа (отсчитывается по часовой стрелке от направления вектора скорости до направления из кормы в нос судна);

$X_{6}, Y_{6}, M_{6}$ продольная и поперечная сила и момент гидродинамического сопротивления на корпусе;

$X_{p}, Y_{p}, M_{p}$ силы, создаваемые обычным рулем;

$T_{E}$ - эффективный упор гребного винта;

$T_{\Pi \text { ע }}, M_{\Pi у}-$ эффективный упор подруливающего устройства и создаваемый им момент;

$X_{\text {внеи }}, Y_{\text {внеш }}, M_{\text {внеш }}$ - силы, обусловленные внешними условиями плавания: ветром, течением, волнением и т. п.;

$k_{11}, k_{22}, k_{66}-$ коэффициенты продольной и поперечной присоединенных масс и присоединенного момента. Каждая из сил и моментов, а также ряд других величин в системе (3) зависят от совокупности факторов $(t, C$, $\left.v(t), w(t), \beta(t), x_{0}(t), y_{0}(t), q(t), U(t), L(t), E(t)\right)$, однако для простоты записи знак такой функциональной зависимости будет опускаться.

В системе (5) предполагается, что компоненты демпфирующих (то есть зависящих от угловой скорости $w$ ) инерционных сил, связанные с присоединенными массами, уже включены в гидродинамические силы сопротивления $\left(X_{6}, Y_{6}, M_{\varepsilon}\right)$ и поэтому явно в системе не прописываются.

Любая из существующих математических моделей включает в себя систему уравнений (5) в полном или упрощенном и огрубленном виде, дополненную совокупностью формул, задающих зависимость всех сил и моментов $\left(X_{6}, Y_{\theta}, M_{6}, X_{p}, Y_{p}, M_{p}, T_{E}, T_{\Pi У}, M_{\Pi У}, X_{\text {внеи }}, Y_{\text {внеи }}, M_{\text {внеш }}\right)$ от факторов $\left(t, C, v(t), w(t), \beta(t), x_{0}(t), y_{0}(t), q(t), U(t), L(t), E(t)\right)$. Именно вид этих формул определяет специфику каждой математической модели.

Рассмотрим теперь динамику водной поверхности, по которой движется судно. Для этого предположим, что водная поверхность является установившимся потоком идеальной жидкости, движущийся без трения. Как и при выводе дифференциальных уравнений равновесия Эйлера, в потоке движущейся жидкости выделяется элементарный параллелепипед и рассматривается равновесие проекций сил на оси координат. Согласно основному правилу динамики, сумма проекций, действующих на элементарный объем, равна произведению массы жидкости на ее ускорение:

$$
\begin{array}{ll}
\text { дляосих } & -\frac{\partial p}{\partial x} d x d y d z=\rho \frac{D w_{x}}{D \tau} d x d y d z, \\
\text { дляоси у } & -\frac{\partial p}{\partial y} d x d y d z=\rho \frac{D w_{y}}{D \tau} d x d y d z, \\
\text { дляоси z } & -\left(\rho q+\frac{\partial p}{\partial x}\right) d x d y d z=\rho \frac{D w_{z}}{D \tau} d x d y d z .
\end{array}
$$


Решив данные уравнения, получим динамическую модель водной поверхности.

В зависимости от характера грунта морского дна, типа судна, глубины и состояния водной поверхности выбираем модели, описывающие динамику рельефа, движение судна и динамику водной поверхности в соответствии с привязкой к местоположению судна. Приведение к единому масштабу по времени и расстоянию обеспечивается путем интерполирования. При этом получится, что карта как бы «дышит», т.е. изобаты постоянно перемещаются в зависимости от высоты и длины волны, как показано на рисунке 1. В этот же момент времени судно по отношению к морскому дну будет отображено на мониторе так, как показано на рисунке 3.

Алгоритм решения данной задачи приведен на рис. 5. На протяжении нескольких лет в устье Дуная, а именно при выходе из Дуная в Черное море проводили повторные многократные наблюдения за изменением рельефа дна, фиксируя при этом в промежутках между наблюдениями, такие гидрометеорологические параметры, как сила и направление ветра, высота и длина волны, а также скорость и направление течения [6]. Пример получаемых результатов после проведения промеров может быть представлен в таком виде как приведено на рисунке $2 a, b$.

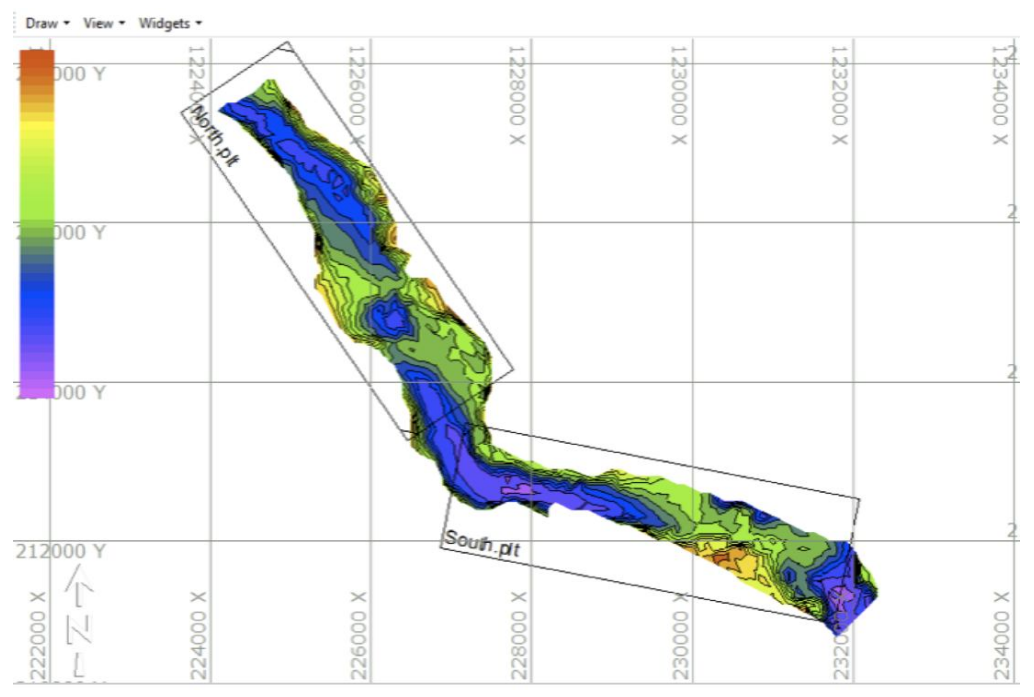

Рис. 2 а. Результаты промера исследуемого участка 
ВІСНИК

ОДЕСЬКОГО НАЦІОНАЛЬНОГО

МОРСЬКОГО УНІВЕРСИТЕТУ

№ 3 (63), 2020
HERALD

OF THE ODESSA NATIONAL

MARITIME UNIVERSITY

№ 3 (63), 2020

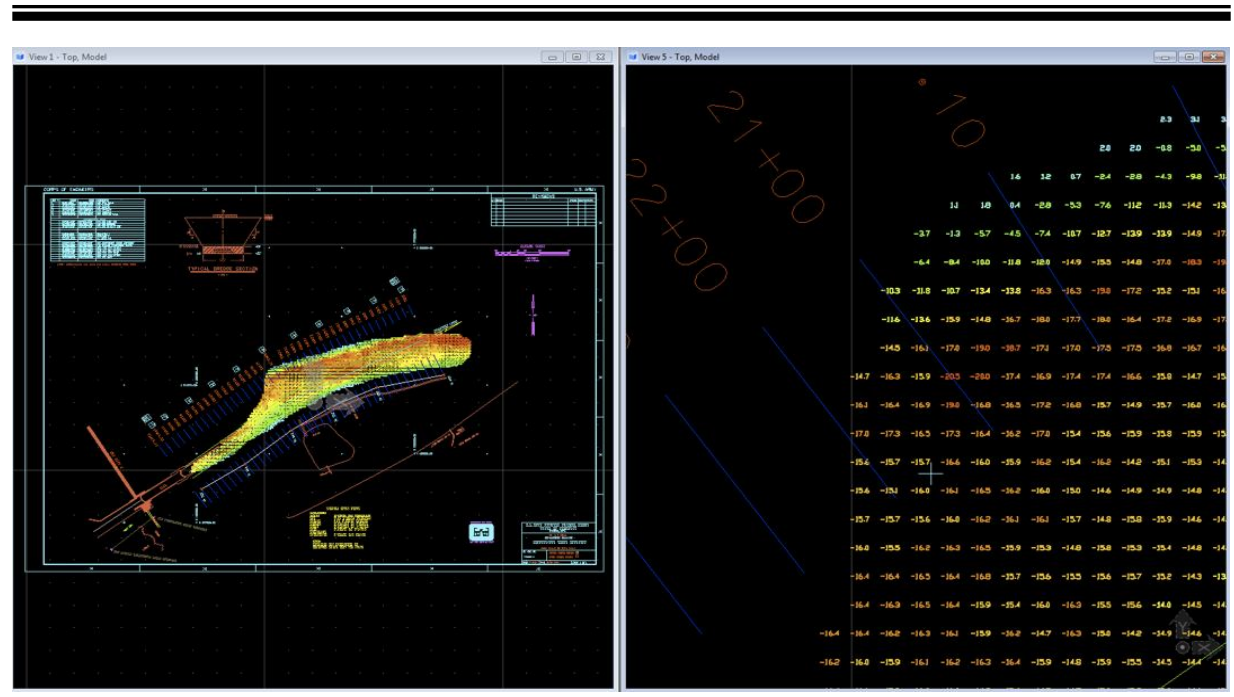

Рис. 2 b. Результаты промера исследуемого участка

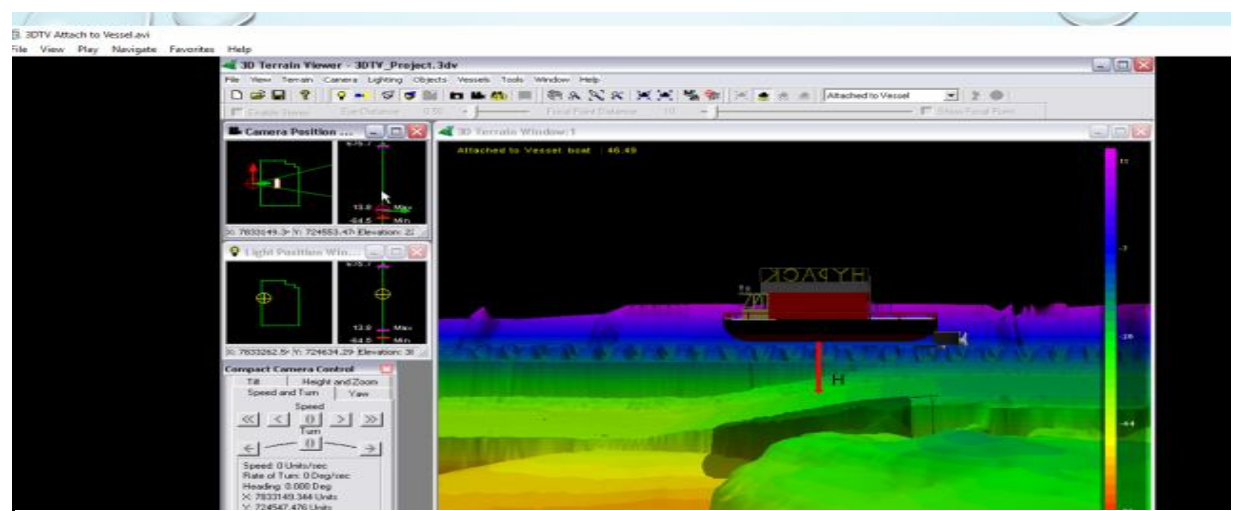

Рис. 3. Динамика судна по отношению к морскомудну

Расписав субстанциональные производные проекций скоростей потока по осям пространственных координат

$$
\begin{aligned}
& \frac{D w_{x}}{D \tau}=\rho\left(\frac{\partial w_{x}}{\partial \tau}+w_{x} \frac{\partial w_{x}}{\partial x}+w_{y} \frac{\partial w_{x}}{\partial y}+w_{z} \frac{\partial w_{x}}{\partial z}\right) \\
& \frac{D w_{y}}{D \tau}=\rho\left(\frac{\partial w_{y}}{\partial \tau}+w_{x} \frac{\partial w_{y}}{\partial x}+w_{y} \frac{\partial w_{y}}{\partial y}+w_{z} \frac{\partial w_{y}}{\partial z}\right) \\
& \frac{D w_{z}}{D \tau}=\rho\left(\frac{\partial w_{z}}{\partial \tau}+w_{x} \frac{\partial w_{z}}{\partial x}+w_{y} \frac{\partial w_{z}}{\partial y}+w_{z} \frac{\partial w_{z}}{\partial z}\right)
\end{aligned}
$$


и, произведя сокращения, получим для соответствующих проекций дифференциальные уравнения жидкости неустановившегося потока

$$
\begin{aligned}
& -\frac{\partial p}{\partial x}=\rho\left(\frac{\partial w_{x}}{\partial \tau}+w_{x} \frac{\partial w_{x}}{\partial x}+w_{y} \frac{\partial w_{x}}{\partial y}+w_{z} \frac{\partial w_{x}}{\partial z}\right) \\
& -\frac{\partial p}{\partial y}=\rho\left(\frac{\partial w_{y}}{\partial \tau}+w_{y} \frac{\partial w_{y}}{\partial y}+w_{x} \frac{\partial w_{y}}{\partial x}+w_{z} \frac{\partial w_{y}}{\partial z}\right) \\
& -\rho q-\frac{\partial p}{\partial z}=\rho\left(\frac{\partial w_{z}}{\partial \tau}+w_{z} \frac{\partial w_{z}}{\partial z}+w_{x} \frac{\partial w_{z}}{\partial x}+w_{y} \frac{\partial w_{z}}{\partial y}\right) .
\end{aligned}
$$

Для установившегося потока $\frac{\partial w_{x}}{\partial \tau}=0, \frac{\partial w_{y}}{\partial \tau}=0, \frac{\partial w_{z}}{\partial \tau}=0$, тогда

$$
\begin{aligned}
& -\frac{\partial p}{\partial x}=\rho\left(w_{x} \frac{\partial w_{x}}{\partial x}+w_{y} \frac{\partial w_{x}}{\partial y}+w_{z} \frac{\partial w_{x}}{\partial z}\right) \\
& -\frac{\partial p}{\partial y}=\rho\left(w_{y} \frac{\partial w_{y}}{\partial y}+w_{x} \frac{\partial w_{y}}{\partial x}+w_{z} \frac{\partial w_{y}}{\partial z}\right) \\
& -\rho q-\frac{\partial p}{\partial z}=\rho\left(w_{z} \frac{\partial w_{z}}{\partial z}+w_{x} \frac{\partial w_{z}}{\partial x}+w_{y} \frac{\partial w_{z}}{\partial y}\right) .
\end{aligned}
$$

Системы уравнений представляют собой дифференциальные уравнения движения идеальной жидкости Эйлера для неустановившегося и установившегося потоков.

При движении вязкой жидкости в потоке, кроме сил давления и тяжести, действуют также силы трения. Для трехмерного потока проекция равнодействующих сил трения на ось $x$ имеет вид

$$
\mu\left(\frac{\partial^{2} w_{x}}{\partial x^{2}}+\frac{\partial^{2} w_{x}}{\partial y^{2}}+\frac{\partial^{2} w_{x}}{\partial z^{2}}\right) d x d y d z=\mu \nabla^{2} w_{x} d x d y d z
$$

Суммы проекций всех сил на оси координат должны быть равны произведению массы жидкости, заключенной в параллелепипеде, на проекции ускорения на оси координат

$$
\begin{aligned}
& \left(-\frac{\partial p}{\partial x}+\mu \nabla^{2} w_{x}\right) d x d y d z=\rho \frac{D w_{x}}{d \tau} d x d y d z ; \\
& \left(-\frac{\partial p}{\partial y}+\mu \nabla^{2} w_{y}\right) d x d y d z=\rho \frac{D w_{y}}{d \tau} d x d y d z ; \\
& \left(p q-\frac{\partial p}{\partial z}+\mu \nabla^{2} w_{z}\right) d x d y d z=\rho \frac{D w_{z}}{d \tau} d x d y d z .
\end{aligned}
$$


После сокращения получим дифференциальные уравнения НавьеСтокса, описывающие движение вязкой капельной жидкости

$$
\begin{aligned}
& \left(-\frac{\partial p}{\partial x}+\mu \nabla^{2} w_{x}\right)=\rho \frac{D w_{x}}{d \tau} ; \\
& \left(-\frac{\partial p}{\partial y}+\mu \nabla^{2} w_{y}\right)=\rho \frac{D w_{y}}{d \tau} ; \\
& \left(p q-\frac{\partial p}{\partial z}+\mu \nabla^{2} w_{z}\right)=\rho \frac{D w_{z}}{d \tau} .
\end{aligned}
$$

Соответствующие субстанциональные производные в уравнениях могут быть выражены как для неустановившегося, так и установившегося течения жидкости.

Решая уравнения (1)-(11) в программной структуре «Matlab», получаем требуемый результат.

Подводя итог данного вопроса, следует отметить, что:

1. В дальнейших исследованиях при использовании разномасштабных данных следует вводить так называемый весовой коэффициент для индексации «scale factor»;

2. В информационный блок навигационных картографических систем ECDIS and Inland ECDIS рекомендуется ввести динамическую составляющую, позволяющую судоводителю видеть положение судна относительно дна в реальном режиме времени;

3. Методы параллельного переноса однообразных форм рельефа рекомендуется применять при волнообразной форме поверхности морского (речного) дна;

4. В данной статье мы рассмотрели только лишь методы параллельного переноса рельефа дна, основанные на данных повторяющихся наблюдений используя методы итераций. В конце нашего эксперимента мы получили расхождение между прогнозируемыми и измеренными значениями координат узловых точек рельефа дна не более $20 \%$.

5. Предложенные модели можно использовать при составлении краткосрочных прогнозов динамики подводного рельефа.

\section{СПИСОК ЛІТЕРАТУРИ}

1. Юдин Ю.И., Сотников И.И. Математические модели плоскопараллельного движения судна. Классификаџия и критический анализ / Ю.И Юдин., И.И Сотников // Вестник МГТУ. 2016. T 9, № 2, C. 200-208. 
ВІСНИК

ОДЕСЬКОГО НАЦІОНАЛЬНОГО

МОРСЬКОГО УНІВЕРСИТЕТУ

№ 3 (63), 2020
HERALD

OF THE ODESSA NATIONAL

MARITIME UNIVERSITY

2. Самонов В.Е. Математическое моделирование движения тонкого слоя жидкости под действием поверхностных сил: дисс. канд.техн.наук: 05.13.18 / В.Е. Самонов. Ставрополь: СГУ. 2013. 145 c.

3. Гладких И.И. Геодезические методы контроля динамики подводного рельефа на участках морских трубопроводов / И.И. Гладких. Одесса: ОГМА, 1997. 153 с.

4. Учитель И.Л., Ярошенко В.Н., Гладких И.И. Основы неогеодинамики / И.Л. Учитель, В.Н. Ярошенко, И.И. Гладких. Одесса: Астропринт. 2000. 144c.

5. Двореикий В.А. Автоматизачия учета радиолокаиионной девиачии / В.А. Двореикий // Судовождение: Сб.научн.трудов / Одесская национальная морская академия. Одесса, 2000. № 2. C. 47-49.

6. Dragomir, P., Rus, T., Avramiuc, N., Dumitru, P. EVRF2007 as Realization of the European Vertical Reference System (EVRS) in Romania, International Symposium GeoCAD08, Alba Iulia, Romania, 09-10 May 2010.

7. Dvoretsky, $V$. The effect of inaccuracies of the auxiliary sensors in the course of data processing of multibeam survey / V.A. Dvoretsky, I.E. Isaac // Bulletin of ONMU. Vip. № 4 (57). Odessa: ONMU Publishing House, 2018. P.160-166.

\section{REFERENCES}

1. Yudin, Yu.I., Sotnykov, Y.Y. Matematycheskye modely ploskoparallelnogo dvyzhenyya sudna. Klassyfykazyya y krytycheskyy analyz [Mathematical models of plane-parallel motion of the vessel. Classification and critical analysis] Westnyk MGTU 2016. T 9, no. 2, pp. 200-208.

2. Samonov, V.E. Matematycheskoe modelyrovanye dvyzhenyya tonkogo sloya zhydkosty pod deystvyem poverhnostnyh syl [Mathematical modeling of the movement of a thin layer of liquid under the action of surface forces]: dyss. k.t.n.: 05.13.18 Stavropol, 2013. $145 \mathrm{p}$.

3. Gladkih, I.I. Geodezycheskye metody kontrolya dynamyky podvodnogo relyefa na uchastkah morskyh truboprovodov. [Geodesic methods for monitoring the dynamics of underwater terrain on sections of offshore pipelines] Odessa OGMA 1997. $153 \mathrm{p}$.

4. Uchytel, Y.L., Yaroshenko, V.N., Gladkih, I.I. Osnovy neogeodynamyky [Fundamentals of neogeodynamic] Odessa 2000. 144 p.

5. Dvoretsky, V.A. Avtomatyzazyya ucheta radyolokazyonnoy deviazyy [Automation of radar deviation accounting], 
ВІСНИК

ОДЕСЬКОГО НАЦІОНАЛЬНОГО

МОРСЬКОГО УНІВЕРСИТЕТУ

№ 3 (63), 2020
HERALD

OF THE ODESSA NATIONAL

MARITIME UNIVERSITY

№ 3 (63), 2020

Sudovozhdenye: Sbornik nauchnyh trudov / ONMA/ Odessa, 2000. no. 2. pp. 47-49.

6. Dragomir, P., Rus, T., Avramiuc, N., Dumitru, P. EVRF2007 as Realization of the European Vertical Reference System (EVRS) in Romania, International Symposium GeoCAD08, Alba Iulia, Romania, 09-10 May 2010.

7. Dvoretsky, $V$. The effect of inaccuracies of the auxiliary sensors in the course of data processing of multibeam survey / V.A. Dvoretsky,, I.E. Isaac // Bulletin of ONMU. Vip. № 4 (57). Odessa: ONMU Publishing House, 2018. P.160-166.

Стаття надійшла до редакиії 20.04.2020

Посилання на статтю: Шпилевский В.В. Использование технологий речных информационных систем для учета динамики рельефа дна// Вісник Одеського національного морського університету: Зб. наук. праць, 2020. № 3(63). C. 106-117. DOI 10.47049/2226-1893-2020-3-106-117.

Article received 20.04.2020

Reference a JournalArtic: Shpylevsky, V. Taking into account the dynamics of the bottom relief for technologies ris // Herald of the Odessa national maritime university. 2020. 3(63), 1O6-117. DOI 10.47049/2226-1893-2020-3-106-117. 